Network Working Group

Request for Comments: 2501

Category: Informational
S. Corson

University of Maryland

J. Macker

Naval Research Laboratory

January 1999

Mobile Ad hoc Networking (MANET):

Routing Protocol Performance Issues and Evaluation Considerations

Status of this Memo

This memo provides information for the Internet community. It does not specify an Internet standard of any kind. Distribution of this memo is unlimited.

Copyright Notice

Copyright (C) The Internet Society (1999). All Rights Reserved.

Abstract

This memo first describes the characteristics of Mobile Ad hoc Networks (MANETs), and their idiosyncrasies with respect to traditional, hardwired packet networks. It then discusses the effect these differences have on the design and evaluation of network control protocols with an emphasis on routing performance evaluation considerations.

1. Introduction

With recent performance advancements in computer and wireless communications technologies, advanced mobile wireless computing is expected to see increasingly widespread use and application, much of which will involve the use of the Internet Protocol (IP) suite. The vision of mobile ad hoc networking is to support robust and efficient operation in mobile wireless networks by incorporating routing functionality into mobile nodes. Such networks are envisioned to have dynamic, sometimes rapidly-changing, random, multihop topologies which are likely composed of relatively bandwidth-constrained wireless links.

Within the Internet community, routing support for mobile hosts is presently being formulated as "mobile IP" technology. This is a technology to support nomadic host "roaming", where a roaming host may be connected through various means to the Internet other than its well known fixed-address domain space. The host may be directly physically connected to the fixed network on a foreign subnet, or be 
connected via a wireless link, dial-up line, etc. Supporting this form of host mobility (or nomadicity) requires address management, protocol interoperability enhancements and the like, but core network functions such as hop-by-hop routing still presently rely upon preexisting routing protocols operating within the fixed network. In contrast, the goal of mobile ad hoc networking is to extend mobility into the realm of autonomous, mobile, wireless domains, where a set of nodes--which may be combined routers and hosts--themselves form the network routing infrastructure in an ad hoc fashion.

2. Applications

The technology of Mobile Ad hoc Networking is somewhat synonymous with Mobile Packet Radio Networking (a term coined via during early military research in the 70's and 80's), Mobile Mesh Networking (a term that appeared in an article in The Economist regarding the structure of future military networks) and Mobile, Multihop, Wireless Networking (perhaps the most accurate term, although a bit cumbersome).

There is current and future need for dynamic ad hoc networking technology. The emerging field of mobile and nomadic computing, with its current emphasis on mobile IP operation, should gradually broaden and require highly-adaptive mobile networking technology to effectively manage multihop, ad hoc network clusters which can operate autonomously or, more than likely, be attached at some point(s) to the fixed Internet.

Some applications of MANET technology could include industrial and commercial applications involving cooperative mobile data exchange. In addition, mesh-based mobile networks can be operated as robust, inexpensive alternatives or enhancements to cell-based mobile network infrastructures. There are also existing and future military networking requirements for robust, IP-compliant data services within mobile wireless communication networks [1]--many of these networks consist of highly-dynamic autonomous topology segments. Also, the developing technologies of "wearable" computing and communications may provide applications for MANET technology. When properly combined with satellite-based information delivery, MANET technology can provide an extremely flexible method for establishing communications for fire/safety/rescue operations or other scenarios requiring rapidly-deployable communications with survivable, efficient dynamic networking. There are likely other applications for MANET technology which are not presently realized or envisioned by the authors. It is, simply put, improved IP-based networking technology for dynamic, autonomous wireless networks. 


\section{Characteristics of MANETs}

A MANET consists of mobile platforms (e.g., a router with multiple hosts and wireless communications devices)--herein simply referred to as "nodes"--which are free to move about arbitrarily. The nodes may be located in or on airplanes, ships, trucks, cars, perhaps even on people or very small devices, and there may be multiple hosts per router. A MANET is an autonomous system of mobile nodes. The system may operate in isolation, or may have gateways to and interface with a fixed network. In the latter operational mode, it is typically envisioned to operate as a "stub" network connecting to a fixed internetwork. Stub networks carry traffic originating at and/or destined for internal nodes, but do not permit exogenous traffic to "transit" through the stub network.

MANET nodes are equipped with wireless transmitters and receivers using antennas which may be omnidirectional (broadcast), highlydirectional (point-to-point), possibly steerable, or some combination thereof. At a given point in time, depending on the nodes' positions and their transmitter and receiver coverage patterns, transmission power levels and co-channel interference levels, a wireless connectivity in the form of a random, multihop graph or "ad hoc" network exists between the nodes. This ad hoc topology may change with time as the nodes move or adjust their transmission and reception parameters.

MANETs have several salient characteristics:

1) Dynamic topologies: Nodes are free to move arbitrarily; thus, the network topology--which is typically multihop--may change randomly and rapidly at unpredictable times, and may consist of both bidirectional and unidirectional links.

2) Bandwidth-constrained, variable capacity links: Wireless links will continue to have significantly lower capacity than their hardwired counterparts. In addition, the realized throughput of wireless communications--after accounting for the effects of multiple access, fading, noise, and interference conditions, etc.--is often much less than a radio's maximum transmission rate.

One effect of the relatively low to moderate link capacities is that congestion is typically the norm rather than the exception, i.e. aggregate application demand will likely approach or exceed network capacity frequently. As the mobile network is often simply an extension of the fixed network infrastructure, mobile ad hoc users will demand similar services. These demands will continue to increase as multimedia computing and collaborative networking applications rise. 
3) Energy-constrained operation: Some or all of the nodes in a MANET may rely on batteries or other exhaustible means for their energy. For these nodes, the most important system design criteria for optimization may be energy conservation.

4) Limited physical security: Mobile wireless networks are generally more prone to physical security threats than are fixedcable nets. The increased possibility of eavesdropping, spoofing, and denial-of-service attacks should be carefully considered. Existing link security techniques are often applied within wireless networks to reduce security threats. As a benefit, the decentralized nature of network control in MANETs provides additional robustness against the single points of failure of more centralized approaches.

In addition, some envisioned networks (e.g. mobile military networks or highway networks) may be relatively large (e.g. tens or hundreds of nodes per routing area). The need for scalability is not unique to MANETS. However, in light of the preceding characteristics, the mechanisms required to achieve scalability likely are.

These characteristics create a set of underlying assumptions and performance concerns for protocol design which extend beyond those guiding the design of routing within the higher-speed, semi-static topology of the fixed Internet.

4. Goals of IETF Mobile Ad Hoc Network (manet) Working Group

The intent of the newly formed IETF manet working group is to develop a peer-to-peer mobile routing capability in a purely mobile, wireless domain. This capability will exist beyond the fixed network (as supported by traditional IP networking) and beyond the one-hop fringe of the fixed network.

The near-term goal of the manet working group is to standardize one (or more) intra-domain unicast routing protocol(s), and related network-layer support technology which:

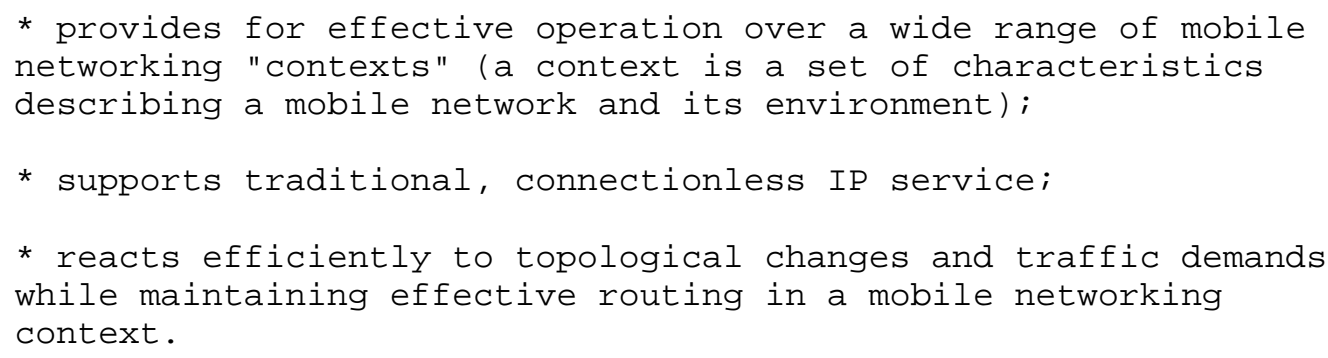


The working group will also consider issues pertaining to addressing, security, and interaction/interfacing with lower and upper layer protocols. In the longer term, the group may look at the issues of layering more advanced mobility services on top of the initial unicast routing developed. These longer term issues will likely include investigating multicast and QoS extensions for a dynamic, mobile area.

\section{IP-Layer Mobile Routing}

An improved mobile routing capability at the IP layer can provide a benefit similar to the intention of the original Internet, viz. "an interoperable internetworking capability over a heterogeneous networking infrastructure". In this case, the infrastructure is wireless, rather than hardwired, consisting of multiple wireless technologies, channel access protocols, etc. Improved IP routing and related networking services provide the glue to preserve the integrity of the mobile internetwork segment in this more dynamic environment.

In other words, a real benefit to using IP-level routing in a MANET is to provide network-level consistency for multihop networks composed of nodes using a *mixture* of physical-layer media; i.e. a mixture of what are commonly thought of as subnet technologies. A MANET node principally consists of a router, which may be physically attached to multiple IP hosts (or IP-addressable devices), which has potentially *multiple* wireless interfaces--each interface using a *different* wireless technology. Thus, a MANET node with interfaces using technologies $A$ and $B$ can communicate with any other MANET node possessing an interface with technology A or B. The multihop connectivity of technology A forms a physical-layer multihop topology, the multihop connectivity of technology B forms *another* physical-layer topology (which may differ from that of $A^{\prime} s$ topology), and the *union* of these topologies forms another topology (in graph theoretic terms--a multigraph), termed the "IP routing fabric", of the MANET. MANET nodes making routing decisions using the IP fabric can intercommunicate using either or both physical-layer topologies simultaneously. As new physical-layer technologies are developed, new device drivers can be written and another physical-layer multihop topology can be seamlessly added to the IP fabric. Likewise, older technologies can easily be dropped. Such is the functionality and architectural flexibility that IP-layer routing can support, which brings with it hardware economies of scale.

The concept of a "node identifier" (separate and apart from the concept of an "interface identifier") is crucial to supporting the multigraph topology of the routing fabric. It is what *unifies* a set of wireless interfaces and identifies them as belonging to the same 
mobile platform. This approach permits maximum flexibility in address assignment. Node identifiers are used at the IP layer for routing computations.

\subsection{Interaction with standard IP Routing}

In the near term, it is currently envisioned that MANETs will function as *stub* networks, meaning that all traffic carried by MANET nodes will either be sourced or sinked within the MANET. Because of bandwidth and possibly power constraints, MANETs are not presently envisioned to function as *transit* networks carrying traffic which enters and then leaves the MANET (although this restriction may be removed by subsequent technology advances). This substantially reduces the amount of route advertisement required for interoperation with the existing fixed Internet. For stub operation, routing interoperability in the near term may be achieved using some combination of mechanisms such as MANET-based anycast and mobile IP. Future interoperability may be achieved using mechanisms other than mobile IP.

Interaction with standard IP Routing will be greatly facilitated by usage of a common MANET addressing approach by all MANET routing protocols. Development of such an approach is underway which permits routing through a multi-technology fabric, permits multiple hosts per router and ensures long-term interoperability through adherence to the IP addressing architecture. Supporting these features appears only to require identifying host and router interfaces with IP addresses, identifying a router with a separate Router ID, and permitting routers to have multiple wired and wireless interfaces.

6. MANET Routing Protocol Performance Issues

To judge the merit of a routing protocol, one needs metrics--both qualitative and quantitative--with which to measure its suitability and performance. These metrics should be *independent* of any given routing protocol.

The following is a list of desirable qualitative properties of MANET routing protocols:

1) Distributed operation: This is an essential property, but it should be stated nonetheless.

2) Loop-freedom: Not required per se in light of certain quantitative measures (i.e. performance criteria), but generally desirable to avoid problems such as worst-case phenomena, e.g. a small fraction of packets spinning around in the network for arbitrary time periods. Ad hoc solutions such as TTL values can 
bound the problem, but a more structured and well-formed approach is generally desirable as it usually leads to better overall performance.

3) Demand-based operation: Instead of assuming an uniform traffic distribution within the network (and maintaining routing between all nodes at all times), let the routing algorithm adapt to the traffic pattern on a demand or need basis. If this is done intelligently, it can utilize network energy and bandwidth resources more efficiently, at the cost of increased route discovery delay.

4) Proactive operation: The flip-side of demand-based operation. In certain contexts, the additional latency demand-based operation incurs may be unacceptable. If bandwidth and energy resources permit, proactive operation is desirable in these contexts.

5) Security: Without some form of network-level or link-layer security, a MANET routing protocol is vulnerable to many forms of attack. It may be relatively simple to snoop network traffic, replay transmissions, manipulate packet headers, and redirect routing messages, within a wireless network without appropriate security provisions. While these concerns exist within wired infrastructures and routing protocols as well, maintaining the "physical" security of of the transmission media is harder in practice with MANETs. Sufficient security protection to prohibit disruption of modification of protocol operation is desired. This may be somewhat orthogonal to any particular routing protocol approach, e.g. through the application of IP security techniques.

6) "Sleep" period operation: As a result of energy conservation, or some other need to be inactive, nodes of a MANET may stop transmitting and/or receiving (even receiving requires power) for arbitrary time periods. A routing protocol should be able to accommodate such sleep periods without overly adverse consequences. This property may require close coupling with the link-layer protocol through a standardized interface.

7) Unidirectional link support: Bidirectional links are typically assumed in the design of routing algorithms, and many algorithms are incapable of functioning properly over unidirectional links. Nevertheless, unidirectional links can and do occur in wireless networks. Oftentimes, a sufficient number of duplex links exist so that usage of unidirectional links is of limited added value. However, in situations where a pair of unidirectional links (in opposite directions) form the only bidirectional connection between two ad hoc regions, the ability to make use of them is valuable. 
The following is a list of quantitative metrics that can be used to assess the performance of any routing protocol.

1) End-to-end data throughput and delay: Statistical measures of data routing performance (e.g., means, variances, distributions) are important. These are the measures of a routing policy's effectiveness--how well it does its job--as measured from the *external* perspective of other policies that make use of routing.

2) Route Acquisition Time: A particular form of *external* endto-end delay measurement--of particular concern with "on demand" routing algorithms--is the time required to establish route(s) when requested.

3) Percentage Out-of-Order Delivery: An external measure of connectionless routing performance of particular interest to transport layer protocols such as TCP which prefer in-order delivery.

4) Efficiency: If data routing effectiveness is the external measure of a policy's performance, efficiency is the *internal* measure of its effectiveness. To achieve a given level of data routing performance, two different policies can expend differing amounts of overhead, depending on their internal efficiency. Protocol efficiency may or may not directly affect data routing performance. If control and data traffic must share the same channel, and the channel's capacity is limited, then excessive control traffic often impacts data routing performance.

It is useful to track several ratios that illuminate the *internal* efficiency of a protocol in doing its job (there may be others that the authors have not considered):

* Average number of data bits transmitted/data bit delivered-this can be thought of as a measure of the bit efficiency of delivering data within the network. Indirectly, it also gives the average hop count taken by data packets.

* Average number of control bits transmitted/data bit delivered--this measures the bit efficiency of the protocol in expending control overhead to delivery data. Note that this should include not only the bits in the routing control packets, but also the bits in the header of the data packets. In other words, anything that is not data is control overhead, and should be counted in the control portion of the algorithm. 
* Average number of control and data packets transmitted/data packet delivered--rather than measuring pure algorithmic efficiency in terms of bit count, this measure tries to capture a protocol's channel access efficiency, as the cost of channel access is high in contention-based link layers.

Also, we must consider the networking *context* in which a protocol's performance is measured. Essential parameters that should be varied include:

1) Network size--measured in the number of nodes

2) Network connectivity--the average degree of a node (i.e. the average number of neighbors of a node)

3) Topological rate of change--the speed with which a network's topology is changing

4) Link capacity--effective link speed measured in bits/second, after accounting for losses due to multiple access, coding, framing, etc.

5) Fraction of unidirectional links--how effectively does a protocol perform as a function of the presence of unidirectional links?

6) Traffic patterns--how effective is a protocol in adapting to non-uniform or bursty traffic patterns?

7) Mobility--when, and under what circumstances, is temporal and spatial topological correlation relevant to the performance of a routing protocol? In these cases, what is the most appropriate model for simulating node mobility in a MANET?

8) Fraction and frequency of sleeping nodes--how does a protocol perform in the presence of sleeping and awakening nodes?

A MANET protocol should function effectively over a wide range of networking contexts--from small, collaborative, ad hoc groups to larger mobile, multihop networks. The preceding discussion of characteristics and evaluation metrics somewhat differentiate MANETs from traditional, hardwired, multihop networks. The wireless networking environment is one of scarcity rather than abundance, wherein bandwidth is relatively limited, and energy may be as well.

In summary, the networking opportunities for MANETs are intriguing and the engineering tradeoffs are many and challenging. A diverse set of performance issues requires new protocols for network control. 
A question which arises is "how should the *goodness of a policy be measured?". To help answer that, we proposed here an outline of protocol evaluation issues that highlight performance metrics that can help promote meaningful comparisons and assessments of protocol performance. It should be recognized that a routing protocol tends to be well-suited for particular network contexts, and less wellsuited for others. In putting forth a description of a protocol, both its *advantages* and *limitations* should be mentioned so that the appropriate networking context(s) for its usage can be identified. These attributes of a protocol can typically be expressed *qualitatively*, e.g., whether the protocol can or cannot support shortest-path routing. Qualitative descriptions of this nature permit broad classification of protocols, and form a basis for more detailed *quantitative* assessments of protocol performance. In future documents, the group may put forth candidate recommendations regarding protocol design for MANETs. The metrics and the philosophy presented within this document are expected to continue to evolve as MANET technology and related efforts mature.

7. Security Considerations

Mobile wireless networks are generally more prone to physical security threats than are fixed, hardwired networks. Existing linklevel security techniques (e.g. encryption) are often applied within wireless networks to reduce these threats. Absent link-level encryption, at the network layer, the most pressing issue is one of inter-router authentication prior to the exchange of network control information. Several levels of authentication ranging from no security (always an option) and simple shared-key approaches, to full public key infrastructure-based authentication mechanisms will be explored by the group. As an adjunct to the working groups efforts, several optional authentication modes may be standardized for use in MANETS.

8. References

[1] Adamson, B., "Tactical Radio Frequency Communication Requirements for IPng", RFC 1677, August 1994. 
Authors' Addresses

M. Scott Corson

Institute for Systems Research

University of Maryland

College Park, MD 20742

Phone: (301) 405-6630

EMail: corsoneisr.umd.edu

Joseph Macker

Information Technology Division

Naval Research Laboratory

Washington, DC 20375

Phone: (202) 767-2001

EMail: macker@itd.nrl.navy.mil 
Full Copyright statement

Copyright (C) The Internet Society (1999). All Rights Reserved.

This document and translations of it may be copied and furnished to others, and derivative works that comment on or otherwise explain it or assist in its implementation may be prepared, copied, published and distributed, in whole or in part, without restriction of any kind, provided that the above copyright notice and this paragraph are included on all such copies and derivative works. However, this document itself may not be modified in any way, such as by removing the copyright notice or references to the Internet society or other Internet organizations, except as needed for the purpose of developing Internet standards in which case the procedures for copyrights defined in the Internet Standards process must be followed, or as required to translate it into languages other than English.

The limited permissions granted above are perpetual and will not be revoked by the Internet society or its successors or assigns.

This document and the information contained herein is provided on an "AS IS" basis and THE INTERNET SOCIETY AND THE INTERNET ENGINEERING TASK FORCE DISCLAIMS ALL WARRANTIES, EXPRESS OR IMPLIED, INCLUDING BUT NOT LIMITED TO ANY WARRANTY THAT THE USE OF THE INFORMATION HEREIN WILL NOT INFRINGE ANY RIGHTS OR ANY IMPLIED WARRANTIES OF MERCHANTABILITY OR FITNESS FOR A PARTICULAR PURPOSE. 